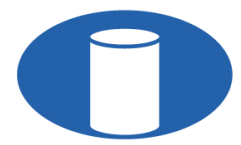

IBRACON Structures and Materials Journal

Revista IBRACON de Estruturas e Materiais

IBRACON

ISSN 1983-4195

ismj.org

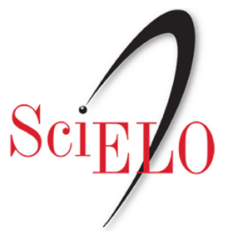

ORIGINAL ARTICLE

\title{
Assessment of low-cost wireless sensors for structural health monitoring applications
}

\section{Avaliação de sensores sem fio de baixo custo para aplicações de monitoramento dinâmico estrutural}

Emerson Toledo Júnior ${ }^{\mathrm{a}}$

Alexandre Cury ${ }^{\mathrm{a}}$ (D)

Jánes Landre Júnior ${ }^{\mathrm{b}}$ (1)

${ }^{a}$ Universidade Federal de Juiz de Fora - UFJF, Programa de Pós-graduação em Engenharia Civil, Juiz de Fora, MG, Brasil

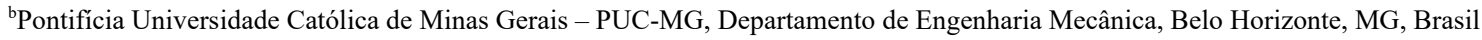

Received 21 April 2020

Accepted 16 July 2020

\begin{abstract}
Structural Health Monitoring (SHM) programs play an essential task in the field of civil engineering, especially for assessing safety conditions involving large structures such as viaducts, bridges, stadiums, and tall buildings. In fact, some of these structures are monitored 24 hours a day, 7 days a week, to supply dynamic measurements that can be used for the identification of structural problems, e.g., presence of cracks, excessive vibration, damage, among others. SHM programs may provide automated assessment of structural health by processing vibration data obtained from sensors attached to the structure. Frequently, SHM uses wired systems, which are usually expensive due to the necessity of continuous maintenance and are not always suitable for sensing remote structures. Conversely, commercial wireless systems often demand high implementation costs. Hence, this paper proposes the use of a low-cost wireless sensing system based on the single board computer Raspberry $\mathrm{Pi}$, which significantly reduces implementation expenses while keeping data's integrity. The wireless communication is performed in real-time through a local wireless network, responsible for sending and receiving vibration data. The proposed system is validated by comparing its results with a commercial wired system through a series of controlled experimental applications. The results suggest that the proposed system is suitable for civil SHM applications.
\end{abstract}

Keywords: structural health monitoring, wireless sensors, low-cost accelerometers.

Resumo: Os programas de Monitoramento Dinâmico Estrutural (SHM, em inglês) desempenham uma tarefa essencial na área da engenharia civil, especialmente para avaliar as condições de segurança que envolvem grandes estruturas, como viadutos, pontes, estádios e edifícios altos. De fato, algumas dessas estruturas são monitoradas 24 horas por dia, 7 dias por semana, com o objetivo de prover medições dinâmicas que possam ser usadas para a identificação de problemas estruturais como, por exemplo, presença de fissuras, vibração excessiva, danos, entre outros. Os programas de SHM podem fornecer uma avaliação automatizada da integridade estrutural, processando dados de vibração obtidos de sensores conectados à estrutura. Frequentemente, o SHM usa sistemas com fio que geralmente são caros devido à necessidade de manutenção contínua e nem sempre são adequados para utilização em estruturas de acesso dificil. Por outro lado, os sistemas sem fio comerciais geralmente exigem altos custos de implementação. Portanto, este artigo propõe o uso de um sistema de monitoramento sem fio de baixo custo baseado no computador de placa única Raspberry Pi que reduz significativamente as despesas de implementação, mantendo a integridade dos dados. A comunicação sem fio é realizada em tempo real através de uma rede local sem fio, responsável pelo envio e recebimento de dados de vibração. O sistema proposto é validado comparando seus resultados aos de um sistema comercial com fio a partir de uma série de aplicações experimentais controladas. Os resultados sugerem que o sistema proposto é adequado para aplicações de SHM em estruturas de engenharia civil.

Palavras-chave: monitoramento dinâmico estrutural, sensores em fio, acelerômetros de baixo custo. applications," Rev. IBRACON Estrut. Mater., vol. 14, no. 2, e14213, 2021, https://doi.org/10.1590/S1983-41952021000200013

Corresponding author: Alexandre Cury. E-mail: alexandre.cury@ufjf.edu.br

Financial support: CNPq (Conselho Nacional de Desenvolvimento Científico e Tecnológico - Project number: 304329/2019-3); FAPEMIG (Fundação de Amparo à Pesquisa do Estado de Minas Gerais - Project number: PPM-00002-16/00001-18), CAPES (Coordenação de Aperfeiçoamento de Pessoal de Nível Superior - Finance Code 001), UFJF (Universidade Federal de Juiz de Fora) and PUC-MG (Pontificia Universidade Catolica of Minas Gerais). Conflict of interest: Nothing to declare. 


\section{INTRODUCTION}

The assessment of vibration tests performed on structural systems has great practical importance for Civil Engineering. Several works published in the literature show different types of structural instrumentation schemes and data acquisition systems. These systems are often used as a tool for dynamic monitoring in structures of significant value, such as the Rio-Niterói Bridge [1] in Brazil, the Z24 Bridge [2] in Switzerland and the Millau Bridge [3] in France.

Numerous researches were carried out using long-term monitoring [4]-[6]. In fact, some structures are monitored 24 hours a day, 7 days a week, to supply dynamic measurements that can be used for the identification of structural problems, such as the presence of cracks [7], [8], excessive vibration [9]-[11], damage identification [12]-[15], among others. Furthermore, structural dynamic analyses allow performing a quite extensive structural evaluation concerning its reliability, vulnerability or even its life cycle [16].

Structural Health Monitoring (SHM) systems may provide automated assessments of structural health by processing data from sensors attached to the structure. SHM often uses wired systems, which are usually expensive due to the necessity of continuous maintenance and are not always suitable for sensing remote structures. Moreover, power and wiring constraints imposed by these systems can increase the acquisition costs of such datasets, impose significant setup delays, and limit the number and location of sensors due to costs and installation logistics. Thus, wireless sensor networks (WSNs) are a possible alternative for structural health monitoring systems, since they enable dense in situ sensing and simplify deployment of instrumentation [17], [18]. Nevertheless, wireless sensor networks should be wisely designed to contemplate important aspects, such as the connection range, i.e., how far is the sensor placed from the data processing unit. For short to mid-range distances, the engineer might opt for Wi-Fi protocols. For long distances, however, radio transmissions could be considered.

Due to their high installation costs, wired sensor networks are generally only feasible for long-term SHM applications. The significant cost reductions of using WSNs for SHM would enable their utilization in important public and private infrastructure and increase the use of applications such as short-term structural monitoring. Such systems could extend the lifespan of numerous structures by enabling earlier damage detection and eliminating the cost of routine inspections [19].

Table 1 gathers the most important comparative metrics for both wired and wireless sensor networks.

Table 1. Comparison of wired and wireless sensor networks (adapted from Noel et al. [19]).

\begin{tabular}{ccc}
\hline Metric & Wired Sensor Networks & Wireless Sensor Networks \\
\hline Cost & Very high, real world examples costing $\$ 10,000$ to $\$ 25,000$ & Low, each sensor node costing approximately $\$ 500$ \\
\hline Deployment Time & Very long, one real world example taking several days & Short, same real world example taking a half hour \\
\hline Lifespan & Long, typically limited by hardware lifespan & Short, typically limited by node battery lifespan \\
\hline Number of Sensors & Typically low due to sensor installation difficulty & Typically higher due to ease of sensor installation \\
\hline Sensor Synchronicity & Very high due to wired connections. & Concern due to wireless connection. \\
\hline
\end{tabular}

The number of studies seeking the development of new sensors for the acquisition of dynamic data is growing continuously. In parallel, one observes the innovation regarding the use of wireless networks, proving better results when it comes to decreasing the presence of noise in signals while reducing maintenance costs. Particularly, WSNs start being used to facilitate data transmission. Wireless acquisition is advantageous for enabling instrumentation in inaccessible places, reducing installation and maintenance costs while expanding its use in situations where large wired systems are not feasible. WSNs have been historically powered by batteries and, as a result, the limiting factor in their overall lifespan has always been the battery lifetime. However, alternative solutions can be listed, such as the use of solar panels and the development of sensors that converted ambient vibrations into electromagnetic energy. Sazonov et al. [20] reported a field test in which the self-powered sensors were used on a rural highway. The sensors were shown to be self-powering even during periods of low traffic.

SHM makes use of different kinds of sensors to monitor structures: displacement sensors, strain-gages, and accelerometers, to name a few. In this paper, we focus on an accelerometer-based system. Accelerometers measure, as the name suggests, accelerations of the surface they are mounted on. From a structural engineering standpoint, accelerometers are characterized by several performance parameters: sensitivity, which denotes the smallest measurable acceleration and is expressed in g's (gravitational acceleration); dynamic range, which denotes the range of accelerations that the device is capable of measuring and is also expressed in g's; and noise, which is measured either as an RMS (root mean square) value, or is expressed as a function of the frequency of vibration [21]. 
Varanis et al. [22] proposed the analysis of mechanical vibrations in the time domain and frequency domain using the Arduino platform. The work has validated its use for educational purposes, and, in their work, they compared the experimental results with those obtained through numerical models using three applications: a cantilever beam, a simply supported beam and a fixed ended beam. The accelerometer used was the MPU-6050 and the results were enough for educational use considering its accuracy and low cost.

Abdelgawad and Yelamarthi [23] presented the importance of the Internet of Things concept for Structural Integrity Monitoring system based on the connectivity of sensors to the worldwide computer network. To this end, it proposes the use of a prototype based on Raspberry Pi 2 for the detection of damage in aluminum plates. The sensor used was not an accelerometer, but a pulse sensor to evaluate damage through the pulse-echo method. The proposed model was able to differentiate the presence of non-existence damage with $0 \%$ error, with $1.03 \%$ error in the location of the damage, and $8.43 \%$ error when measuring the damage. However, results of the proposed prototype were not compared to the conventional accelerometer counterpart.

Afsana et al. [24] proposed a wireless sensor network for detecting cracks in concrete structures. For this, they suggested the use of Raspberry Pi 3 Model B along with an unspecified vibration sensor. A relevant feature is the use of a GSM modem to perform the remote communication with the user, uploading the data on a web page. The work has no test results and is based on the indication of hardware for assembly of a prototype. A couple of works also pointed in this direction, such as Shachi and Manjunatha [25] and Chandankhede [26].

In this sense, this paper presents a study to explore the potentialities and verify the suitability of low-cost sensors as replacements to commercial wired accelerometers in specific testing scenarios. To this end, this paper details the design, programming, implementation, and evaluation of a low-cost wireless accelerometer based on a small singleboard computer named Raspberry Pi. This platform significantly reduces implementation costs while keeping data's integrity. The wireless communication is performed in real-time through a local wireless network, responsible for sending and receiving data. To get acceleration data, two sensors are used in this work and are compared to evaluate the cost-effectiveness of each; these are the MPU-6050 and the MPU-9250. The proposed system is validated by comparing its results with a commercial wired system through benchmark experimental applications performed in laboratory.

\section{DESIGN AND IMPLEMENTATION OF THE SYSTEM}

In order to design a reliable and robust wireless accelerometer, certain minimum prerequisites are needed to fulfil an adequate sensing performance. The most important requirements are: sensor's autonomy, real-time data acquisition and transmission, low cost, and data's reliability.

Concerning sensor's autonomy, the choice of proper physical components plays a pivotal role. Thus, using modules with low-power consumption and a platform that supports the use of an external battery and sustainable energy systems are essential.

For a SHM system, the device must be able to receive orders from the remote user and send real-time data acquisition according to a given command. That means real-time data acquisition and transmission. Being low cost is the main purpose of this work, proposing an inexpensive wireless system. Thus, the physical components should be easy to buy, affordable as well as the controlling software should be preferably free.

Within this study, the synchronization is made using a broadcast command without addressing a specific IP. This means that a packet is received by all equipment almost simultaneously. Given the fact that all equipment is connected to a local network and care was taken to avoid interference from nearby networks, the latency reached was $1-2 \mathrm{~ms}$, which is not sufficient to significantly offset the frequency range proposed for the system.

For large-scaled applications, one could easily use GPS. However, it should be kept in mind that increasing the complexity of Wi-Fi networks, it would also increase the communications' latency. Thus, it would be possible to easily use a GPS module that has microsecond accuracy for remote synchronization. Such a procedure is already widely used for standalone sensors that require a high level of accuracy.

Data's reliability covers several factors such as noise, data loss, sensitivity, frequency range and duration of data acquisition. Noise can vary according to the accelerometer's resolution and to its way of acquiring data. However, it can also be influenced by how devices are connected to each other, especially the wiring. Data loss happens especially in wireless communication if the wireless accelerometers and the data acquisition module are not well synchronized or if there is an incompatibility between the devices. Sensitivity, frequency range and duration must be provided for each application so that the accelerometer is arranged to receive values in a frequency band. Thus, sampling frequency and time of acquisition are defined according to the sampling Nyquist-Shannon theorem. The proposed wireless system can be schematically represented as Figure 1 shows. 


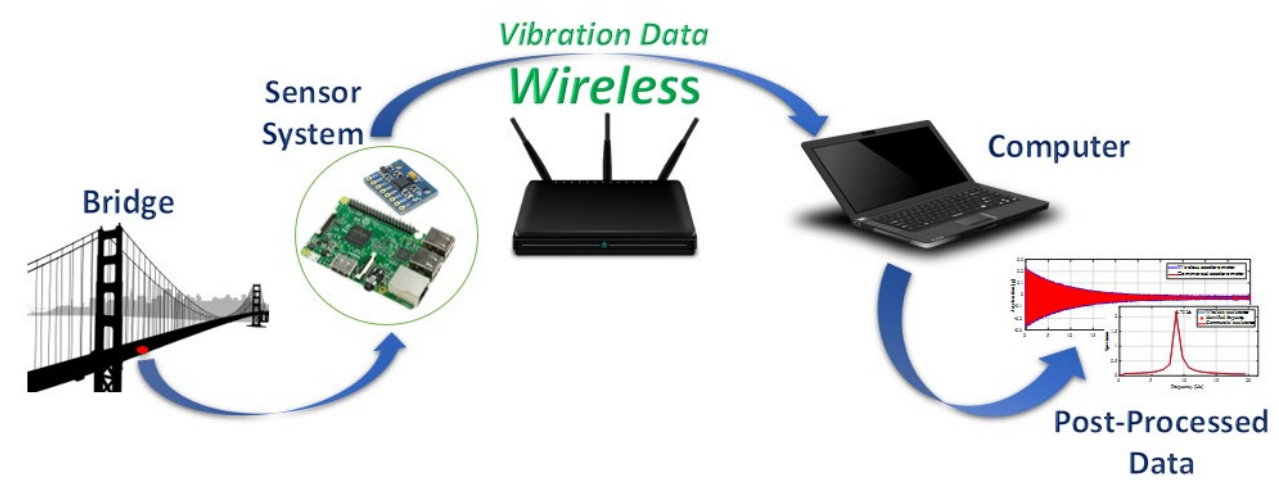

Figure 1. Wireless system schematics.

\section{HARDWARE}

The physical components are chosen to meet the aforementioned prerequisites. Their specifications should meet design specifications and must be compatible with each other. To assemble the wireless system proposed in this work, some components are used, including primarily the Raspberry Pi 3 Model B, followed by the sensors MPU-6050 and MPU-9050.

\subsection{Raspberry Pi 3 Model B}

Raspberry Pi is known as a single-board computer, being a complete computer built on a single circuit board, with microprocessor, memory, input/output $(\mathrm{I} / \mathrm{O})$ and other features required for a functional computer.

Raspberry Pi 3 Model B shown in Figure 2 is based on a Quad Core 1.2GHz Broadcom BCM2837 64bit and 1GB RAM. Its connectivity is made by cable with $10 / 100 \mathrm{Mbit} / \mathrm{s}$ Ethernet or wirelessly via BCM43438 wireless LAN and Bluetooth Low Energy (BLE) on board. The design of this model does not include non-volatile memory - such as a hard drive - but it has an SD card slot for data storage, which is a must for the proposed prototype.

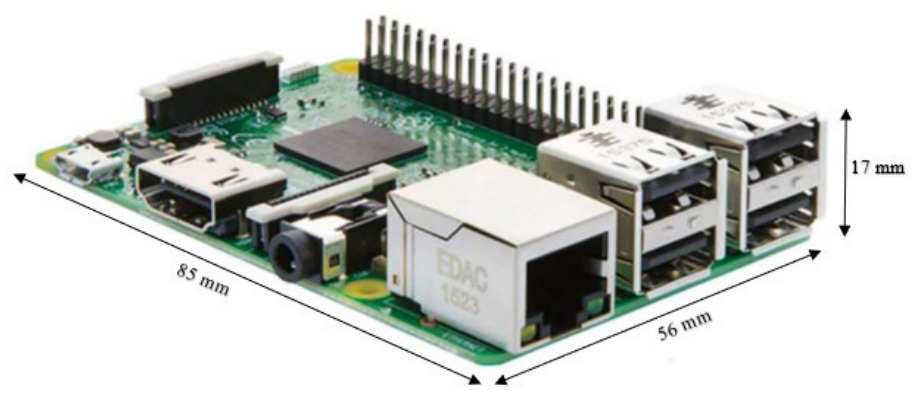

Figure 2. Raspberry Pi 3

\subsection{MPU-6050}

The InvenSense MPU-6050 sensor contains a MEMS accelerometer and a MEMS gyro on a single chip. It is very accurate since it contains 16-bit analog-to-digital conversion hardware for each channel. Therefore, it captures the $\mathrm{x}, \mathrm{y}$, and $\mathrm{z}$ directions at the same time. The sensor uses the $\mathrm{I} 2 \mathrm{C}$-bus to interface with Raspberry Pi. MPU-6050 was the first MotionTracking device designed for low-power, low-cost, and high-performance requirements for smartphones, tablets, and handheld sensors. All this in a device of dimensions $4 \mathrm{~mm} \times 4 \mathrm{~mm} \times 0.9 \mathrm{~mm}$ with prices ranging from around 6 to 8 US dollars.

The accelerometer is programmable by the user in several acceleration ranges, being able to be set on $\pm 2 \mathrm{~g}, \pm 4 \mathrm{~g}, \pm 8 \mathrm{~g}$ and $\pm 16 \mathrm{~g}$. The MPU-6050 has an input voltage of 6-16V, a low-pass filter between 5-260Hz, and a data output rate between 4 to $1000 \mathrm{~Hz}$. The prototype developed in this work uses the accelerometer sensor set on frequency range of $\pm 2 \mathrm{~g}$. 


\subsection{MPU-9250}

The InvenSense MPU-9250 sensor is also a sensor containing a MEMS accelerometer and a MEMS gyro on a single chip. Its specifications are remarkably similar to the MPU-6050 previously mentioned, but with improvements that can be useful in structural monitoring.

From all improvements, three are highlighted: i) the promise of a $25 \%$ reduction in noise; ii) the increase of the sampling rate, to $4000 \mathrm{~Hz}$ (versus $1000 \mathrm{~Hz}$ of the MPU-6050); and iii) the data output rate is between 2.5 to 1000 Hz. This allows sampling in the 3 axes simultaneously at approximately $1300 \mathrm{~Hz}$, while its predecessor was limited to approximately $300 \mathrm{~Hz}$ if the 3 axes were acquired at the same time.

According to the Nyquist-Shannon Theorem, a bandlimited continuous-time signal can be sampled and perfectly reconstructed from its samples if the waveform is sampled over twice as fast as its highest frequency component. Thus, for the case of the MPU-6050, it would be possible to properly sample a signal containing frequencies up to $150 \mathrm{~Hz}$, whereas for the MPU-9250 such value would increase to $1300 \mathrm{~Hz}$.

For common civil structures, which are the main focus of this paper, both would be appropriate for use. However, it is very important to have vibration signals sampled with adequate time and frequency resolutions (i.e., more data samples within a time window), since this would allow a better representation of the structure's dynamic behavior, yielding better post-processing of the data (e.g. modal identification, filtering, input to damage detection techniques, etc.).

\section{SOFTWARE}

The choice of suitable software to program and control the prototype is extremely important, since one of the most important points of the prototype is to efficiently perform the communication between the remote user and the accelerometer. In addition, considering a low-cost system, preference was given to free distribution and open source software. Concerning Raspberry Pi, it is still crucial to carefully choose the operating system, which will be decisive for the suitable functioning of the equipment according to the needs of the sensing system.

\subsection{Raspbian Lite}

Raspbian [27] is a free operating system based on Debian and optimized for Raspberry Pi hardware. The operating system is composed of a set of basic programs and utilities that allow the initialization and basic use of the device. Its reputation revolves around being a high-quality, stable, and scalable operating system. Being scalable indicates the ability of the system to increase its performance according to the increase of resources, for example, more powerful hardware.

Because Raspbian is an adaptation of Debian, there is a wide range of bibliography and documentation, as both have been extensively explored by users of various applications. The Raspbian operating system itself is offered through a graphical interface. The use of the graphical interface ends up generating a computational cost that does not generate return to the remote sensing system. Thus, it is interesting that the equipment has as priority only the acquisition and processing of the acceleration data.

From this comes the Raspbian Lite operating system, being a variation of the Raspbian system with the same functionalities, but without the graphical interface. All the configuration of the device, as well as its use and monitoring, is performed through the system terminal from command lines. With this, the prototype processor is relieved of graphic processing and can be used exclusively on its function as a sensor.

\subsection{Putty}

Putty [28] is an SSH communication client originally developed by Simon Tatham, an open source program that is currently developed and enhanced by a group of volunteers. Its utility is to be able to remotely access the terminal of any Raspberry Pi that is connected in the same Wi-Fi network as the user. That is, the user can reprogram any system sensor remotely, having access to the terminal of the coordinator of the accelerometer, in this case, the Raspberry Pi.

$\mathrm{SSH}$ is an acronym for Secure Shell which is a cryptographic network tool used in insecure networks to operate safely without data interception. The main use of this tool is the remote access of computers in a network, having access to their terminal. 


\subsection{WinSCP}

WinSCP [29] is a software that is designed to be a free solution for file transfers using the FTP protocol and its variants, being a free award-winning software for file management. In the wireless sensing system, the accelerometers work remotely. This means that the computer does not have to be connected to the system during the test. Once automated, the system will run until the user asks to stop or set an end-of-trial parameter.

During the test, the accelerometers can instantly send their readings or save the dataset to their flash memory. Thus, WinSCP arises to enable the process of transferring files safely between the remote sensor and the user.

FTP is an acronym for File Transfer Protocol which is a standard network protocol used to transfer digital files between client and server on a computer network. This protocol is not considered secure purely. Thus, the variation used in this work is SFTP, which is the FTP protocol with SSH authentication explained previously.

To connect to the sensors and to recover the acceleration data, it suffices that the user is connected to the same Wi-Fi network and knows the IP address of the sensor. It is also possible to set a password authentication before full accessing Raspberry Pi.

\section{EXPERIMENTAL APPLICATIONS}

The effectiveness and robustness of the proposed wireless acquisition system is assessed through a series of experimental applications. The methodology relies on attaching three different accelerometers to two excitation modules. The first accelerometer is the CCLD 4507B, a commercial accelerometer with calibration certificate connected to a QuantumX MX1601B acquisition system; this setup represents a traditional wired instrumentation and is used as baseline for comparison. This assembly costs around 3000 US dollars.

The second accelerometer is the MPU-6050 connected to a Raspberry Pi 3 Model B, being a basic proposal of prototype using Raspberry Pi. The third accelerometer is the MPU-9250 connected to a Raspberry Pi 3 Model B, being a suggestion of improvement of the MPU-6050. Both assemblies cost around 30 US dollars.

The communication of the first accelerometer is entirely done through cables, whereas for the second and third accelerometers, data transmission is performed via local Wi-Fi network established in laboratory without connection to the Internet. All tests are performed at a constant acquisition rate of $600 \mathrm{~Hz}$, considering the Nyquist-Shannon theorem that limits the tested frequencies at $300 \mathrm{~Hz}$. Two sets of tests are performed separately using two different shakers.

The first testing setup consists in an experiment performed using an accelerometer calibrator. This equipment can generate a pure sinusoidal wave without harmonics in a frequency and amplitude predefined during its manufacture. The model PCB $394 \mathrm{C} 06$ operates at a frequency of $159.2 \mathrm{~Hz}$ with a peak acceleration of $1.00 \mathrm{~g}\left(9.81 \mathrm{~m} / \mathrm{s}^{2}\right)$. In this case, since there is a constancy of frequency and amplitude of acceleration, tests with three simultaneous accelerometers are not mandatory and thus, can be performed one after another, consecutively. Figure 3 shows the instrumentation scheme for these tests.

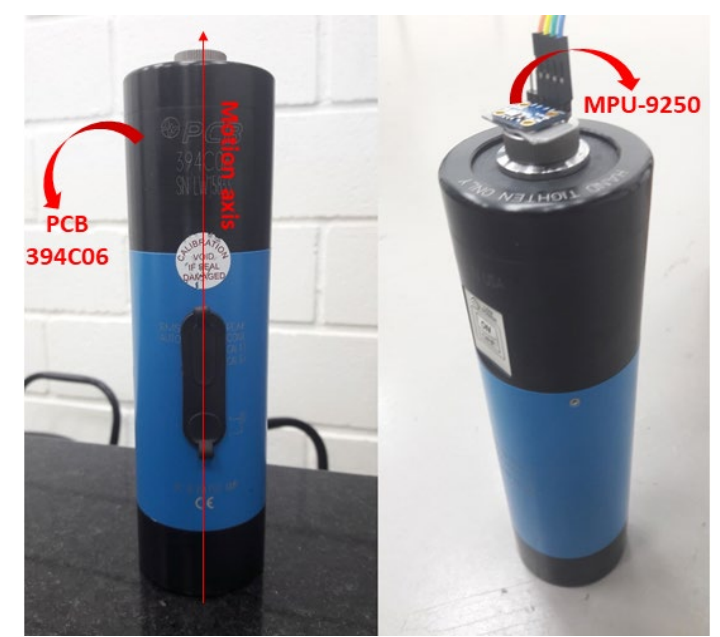

Figure 3. Testing setup (MPU-6050 attached to the calibrator).

The second testing setup consists in a modulated experiment using a ModalShop 2004E shaker. To modulate frequency and amplitude, a Keysight 33210A arbitrary waveform and function generator was used. The generator chosen is digital to offer greater control and stability to the system. In this process, the acceleration data are registered simultaneously for all three accelerometers and then compared. Figure 4 shows the instrumentation scheme for these tests. 


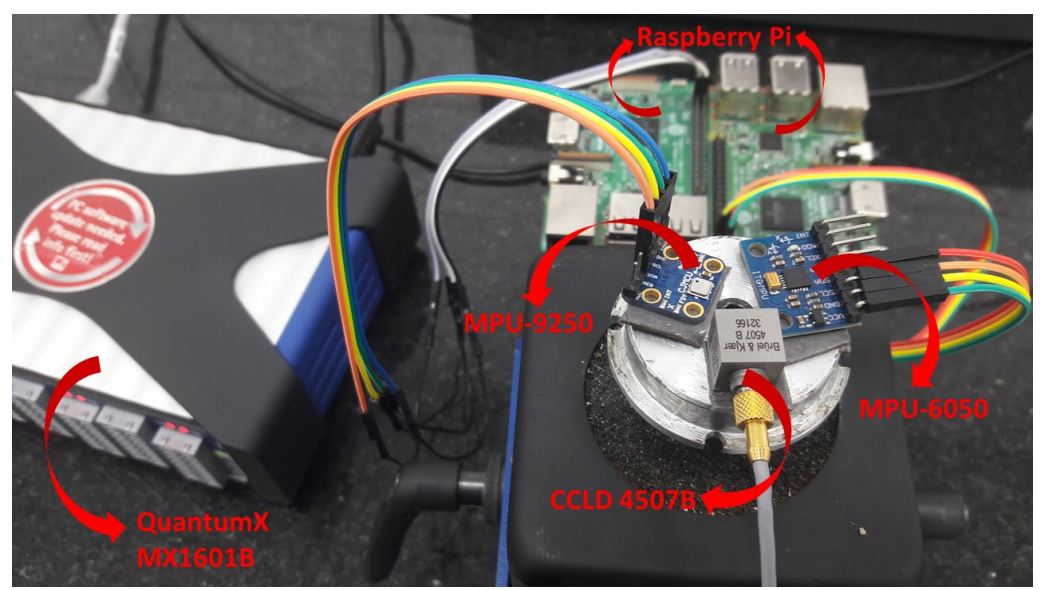

Figure 4. Testing setup (three accelerometers attached to the shaker).

\subsection{Prototype Implementation}

The process starts with the setup of a local Wi-Fi network, choosing the best channel to avoid interference during wireless communication.

Then, Raspbian Lite operating system is installed in the Raspberry PI module, setting the configuration for automatic connection to the local network with access password and the enabling of the SSH and I2C interfaces. Thus, tools such as I2C Tools and Python SMBUS are installed, followed by the algorithm that controls the data acquisition process, e.g. sampling rate, beginning of test, end of test, method of internal storage, data transferring and so on.

In this methodology, the start parameter of each experimental test is a command sent by a computer to all the equipment connected to the same network. After receiving this command, all prototypes start registering the readings at $600 \mathrm{~Hz}$, with the end of the test being its duration (set to 90 seconds). Data is stored in the memory card in txt format.

Acquired data can be internally post-processed by the prototype using the Raspberry Pi's processing capability. However, this work is mainly interested in analyzing the data without any internal treatment. Thus, the txt files are stored until the user remotely accesses them and downloads them. Files are identified by their start acquisition date and time, with an accuracy of seconds. Figure 5 schematizes the entire instrumentation process.

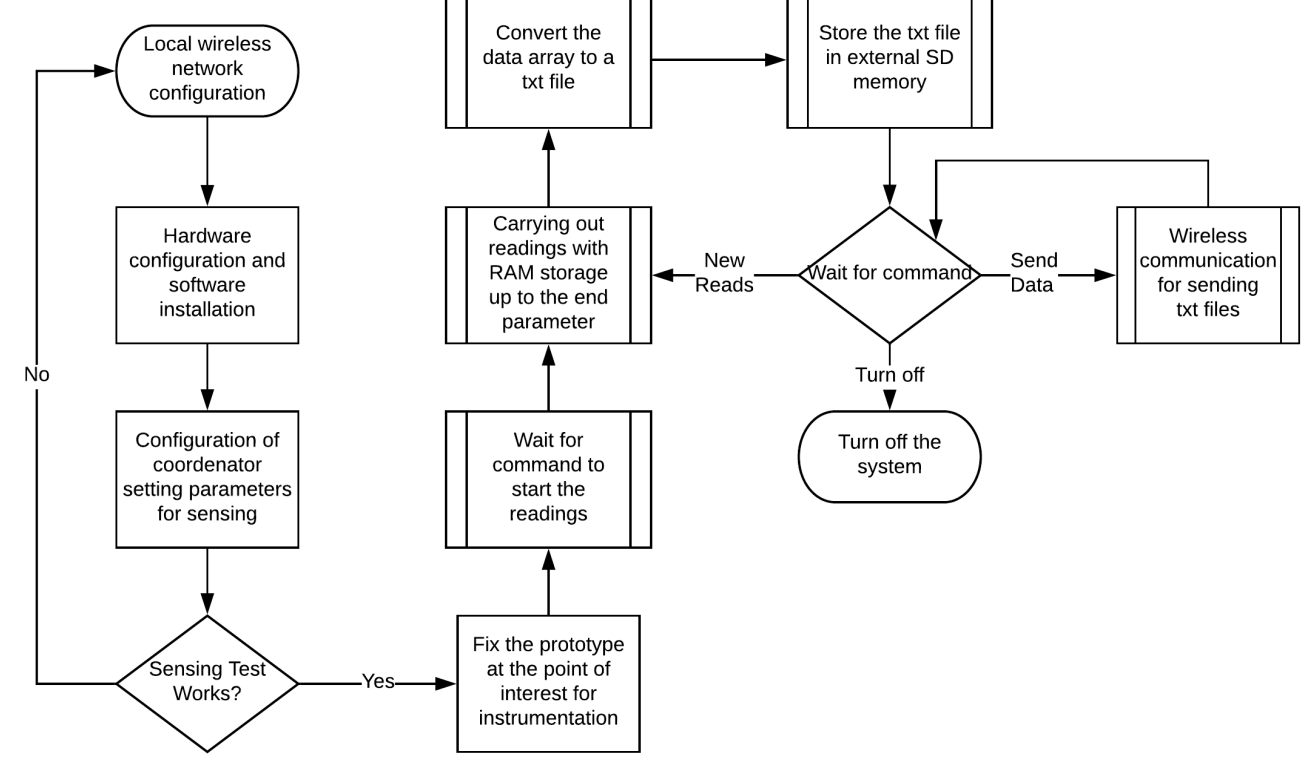

Figure 5. Flowchart of the prototype's implementation 


\subsection{Zero-G output tests}

The zero-g output tests were performed comparing the response of the three accelerometers placed on a flat surface at rest without any vibration, as a way of assessing internal noise. Figure 6 depicts a part of such tests.

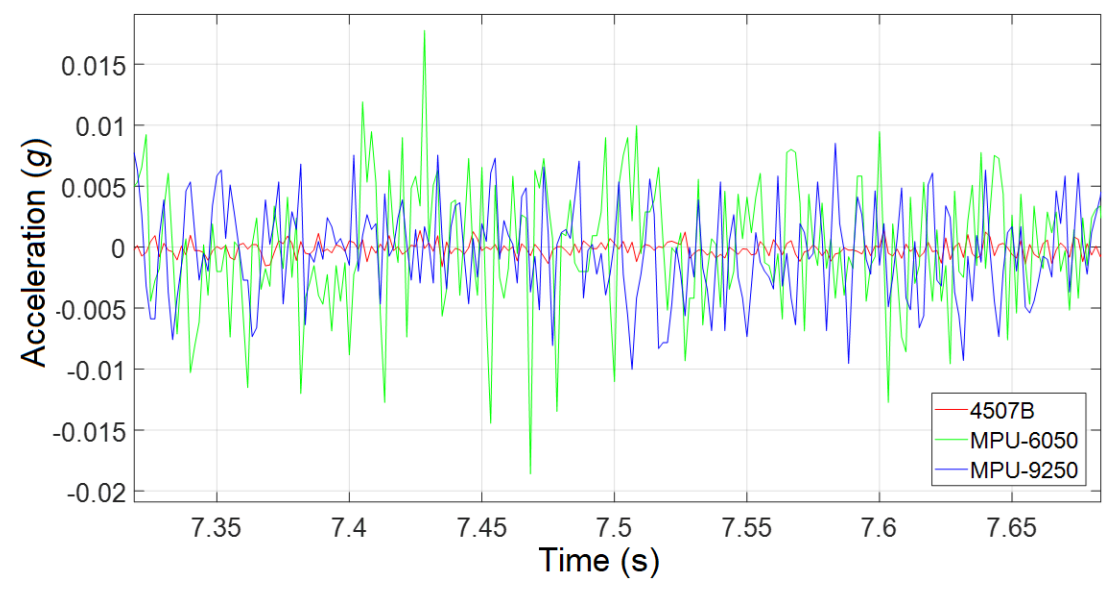

Figure 6. Comparison of the responses registered by the accelerometers at rest.

These results represent a limitation of the proposed system concerning vibration tests that generally occur under very low acceleration amplitudes (ambient excitation). However, this drawback is circumvented in frequency domain analyses, where the low cost sensors present very good results compared to the commercial accelerometer (further discussion is provided in section 5.3).

To better compare the influence of internal noise of each accelerometer, a statistical analysis is now proposed. When analyzing the noise range of the three accelerometers, $5 \%$ of the readings that are too far away from the mean values outliers - are removed to avoid measurements that do not represent the usual behavior of the accelerometers. Thus, the $95 \%$ of the retained readings represent a range of noise that the accelerometer shows without external vibration. These acceleration ranges are shown in Table 2.

Table 2. Comparison of noise bands.

\begin{tabular}{cccc}
\hline & \multicolumn{3}{c}{ Zero-G output (g) } \\
\cline { 2 - 4 } & Negative peak & Positive peak & Average range \\
\hline 4507B & $-0,0014$ & 0,0013 & $\pm 0,00135$ \\
\hline MPU-6050 & $-0,0105$ & 0,0100 & $\pm 0,01025$ \\
\hline MPU-9250 & $-0,0095$ & 0,0095 & $\pm 0,00950$ \\
\hline
\end{tabular}

According to Table 2, the noise band for the MPU-6050 is 759\% larger than the 4507B's counterpart, while the MPU-9250 is 704\% larger. Thus, there is a noise reduction of $7.3 \%$ from the MPU-9250 to its predecessor.

Even though the percentage differences are substantial, the absolute differences may be insignificant depending on the desired application for the sensors. If the application requires a sensitivity of $0.001 \mathrm{~g}$, the commercial accelerometer would be the most recommended. On the other hand, applications that allow an uncertainty of $0.01 \mathrm{~g}$ in the measurements, both MPU-6050 and MPU-9250 could meet the requirements of resting noise at a much lower cost.

The noise at rest does not actually represent the total existing reading error when the structure is in acceleration, but rather from which minimum acceleration of the structure it is possible to differentiate what is real structural acceleration to what is resting noise.

\subsection{Frequency domain analyses}

In the frequency domain, the interest is in determining the precision and accuracy of the results obtained by the prototypes when compared to the commercial accelerometer. 
To this end, the first analysis is performed using the universal calibrator. This equipment guarantees an excitation frequency equal to 159,2 Hz. The second analysis utilizes the ModalShop 2004E shaker of modulated frequency and amplitude.

Figure 7 shows the frequency responses of the three accelerometers using the universal calibrator. For all cases, the natural frequency is exactly identified at $159.2 \mathrm{~Hz}$. The difference in the peak values in the y axis reflects the divergences in the time domain, which will be discussed in the next section. Other identified frequencies indicated by the arrows were found only by the MPU-6050 and MPU-9250, with amplitudes proportional to the noise detected in the signals. Such spurious frequencies are not representative, given the discrepancy of their amplitudes in relation to main frequencies.

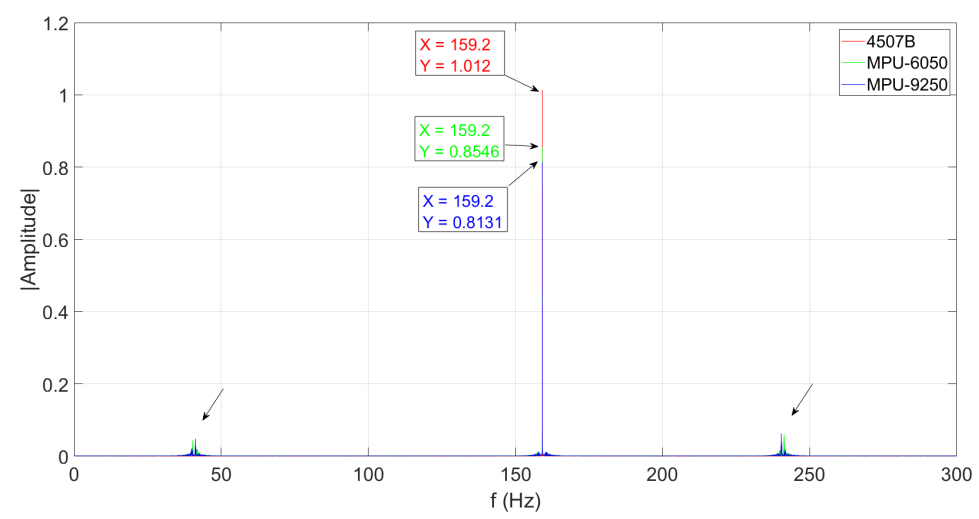

Figure 7. Responses in the frequency domain using the universal calibrator at $159,2 \mathrm{~Hz} / 1,0 \mathrm{~g}$.

Figure 8 shows the results in the frequency domain for the tests carried out at $2.5 \mathrm{~Hz} / 0,02 \mathrm{~g}$. This represents the 'worst' scenario for the prototypes since it has the lowest signal-to-noise ratio. For this case, all frequencies identified by the 4507B were also detected by the other two low cost accelerometers. It is interesting to note the tendency to find multiple frequencies from $2.5 \mathrm{~Hz}$ to the $95 \mathrm{~Hz}$ frequency, except for a few that were not detected by any of the three accelerometers.

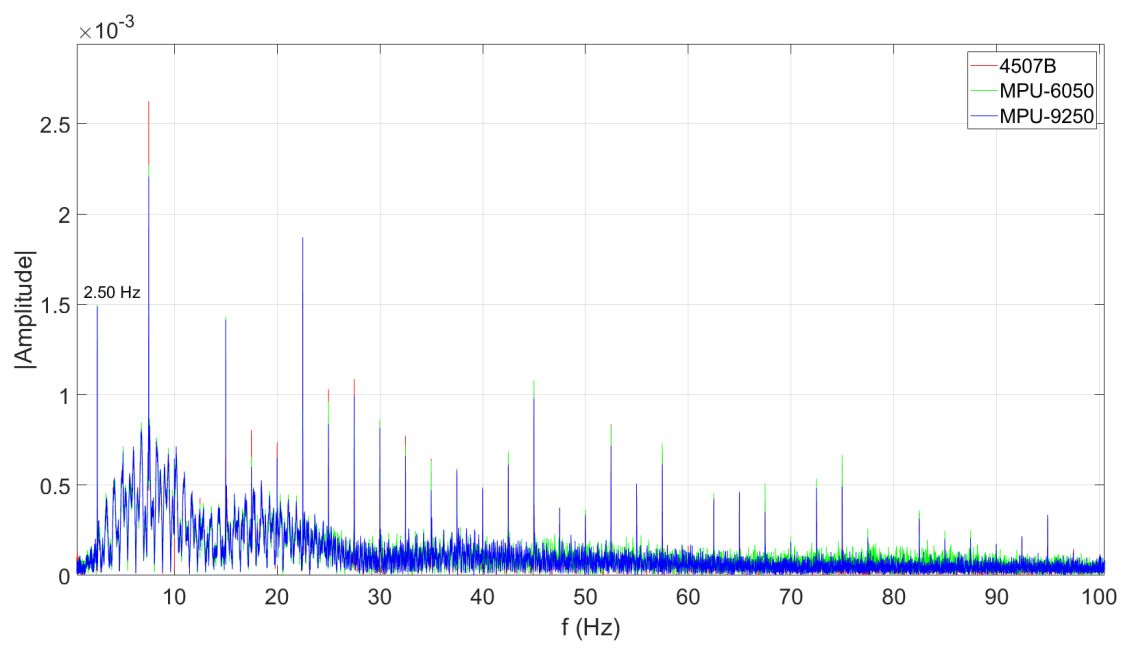

Figure 8. Responses in the frequency domain using the modulated shaker at $2,5 \mathrm{~Hz} / 0,02 \mathrm{~g}$.

For the frequency of $20 \mathrm{~Hz}$, the most unfavorable situation is the acceleration amplitude of $0.2 \mathrm{~g}$ (see Figure 9). Again, the prototypes presented very satisfactory results. 


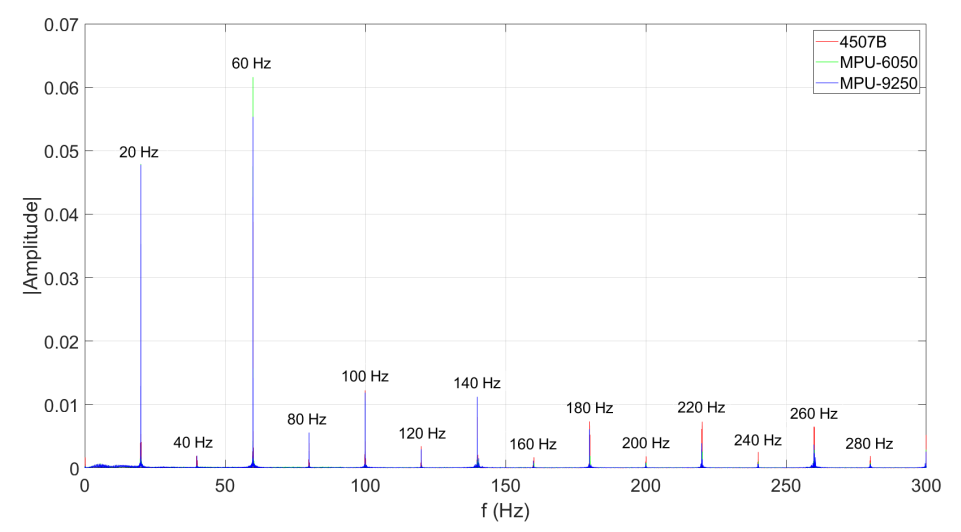

Figure 9. Responses in the frequency domain using the modulated shaker at $20 \mathrm{~Hz} / 0,2 \mathrm{~g}$.

The accuracy, precision and reliability of the results obtained by the prototypes allow validating their operation in the frequency domain within the frequencies and amplitudes tested.

\subsection{Time domain analyses}

The main consequence of high internal noise levels lies mostly in the time domain. Therefore, quantifying the prototype's divergences from the commercial accelerometer is crucial for assessing their potential to accurately represent structural vibration. Hence, quantifying such errors allows establishing confidence acceleration intervals for the prototypes. While within these confidence intervals, the prototypes can be used in structural diagnostics established from temporal data, such as the criterion of maximum acceleration in a structure.

The first testing setup uses the universal accelerometer calibrator. The acceleration amplitude of $1,0 \mathrm{~g}$ without harmonic frequencies is guaranteed by certification, being the only test with a pure sinusoidal behavior. Results for the three accelerometers are shown in Figure 10.

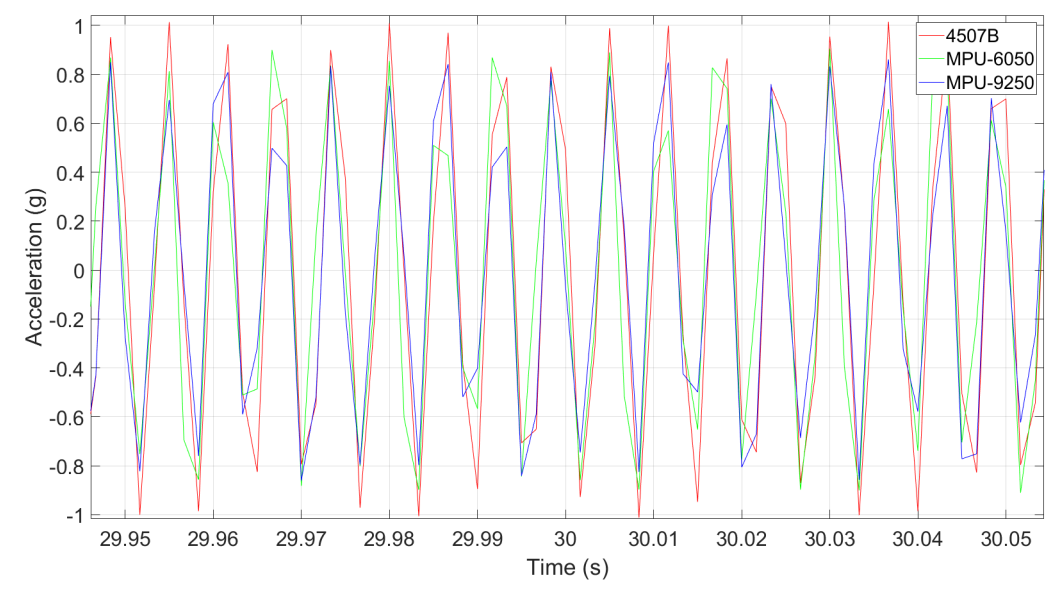

Figure 10. Responses using the universal calibrator at $159,2 \mathrm{~Hz} / 1,0 \mathrm{~g}$.

By observing Figure 10, both MPU-6050 and MPU-9250 were able to reasonably represent the behavior of the vibration in the time domain when compared to the 4507B. Nonetheless, since the sampling rate is $600 \mathrm{~Hz}$ and the excitation frequency is $159.2 \mathrm{~Hz}$, the acceleration peaks in the readings often do not match the accelerator peaks of the calibrator. Table 3 shows the maximum and minimum accelerations found for each accelerometer. 
Table 3. Absolute acceleration peaks using the calibrator.

\begin{tabular}{cccc}
\hline & & Acceleration (g) & \\
\cline { 2 - 4 } & Negative peak & Positive peak & Average range \\
\hline 4507B & $-1,0279$ & 1,0191 & $\pm 1,0235$ \\
\hline MPU-6050 & $-0,9717$ & 0,9600 & $\pm 0,9658$ \\
MPU-9250 & $-0,8671$ & 0,8717 & $\pm 0,8694$ \\
\hline
\end{tabular}

The maximum acceleration values are important to realize that the 4507B, which has the lowest noise level, obtained a higher acceleration range value based on the maximum readings. Therefore, it is necessary to establish a multiplication factor for the prototype accelerometers, just as the 4507B has a calibration constant in its certificate.

The multiplication factor is evaluated by firstly identifying the positions in the time in which the dominant frequency reaches its maximum acceleration and then by extracting its respective acceleration peaks.

For the universal calibrator, the multiplication factor's evaluation becomes simpler because the signal has only one frequency. In the modulated shaker, however, it is necessary to use a frequency filter to obtain the time location where the maximum acceleration for the dominant frequency is, and then return to the raw signal to obtain the acceleration at that instant of time, since the frequency filters change the signal amplitude. Next, a filter is created to remove outliers. Thus, $5 \%$ of the most distant readings from the mean value are removed, i.e. $2.5 \%$ of the lowest readings and $2.5 \%$ of the highest readings.

With the average of the maximum values already filtered, all accelerometers are then compared. Note that for each set of experiments, new multiplication factors must be evaluated. Tables 4 and 5 show the average values (and respective standard-deviations) of the multiplication factors for the tests performed. Tests were repeated five times for each combination 'acceleration band' $\mathrm{x}$ 'frequency'.

Table 4. Multiplication Factors for the MPU-6050 (average and standard-deviation values).

\begin{tabular}{|c|c|c|c|c|c|c|c|c|}
\hline & & \multicolumn{7}{|c|}{ Acceleration band } \\
\hline & & $0,02 \mathrm{~g}$ & $0,05 \mathrm{~g}$ & $0,1 \mathrm{~g}$ & $0,2 \mathrm{~g}$ & $0,5 \mathrm{~g}$ & $1 \mathrm{~g}$ & $1,5 \mathrm{~g}$ \\
\hline \multirow{7}{*}{ 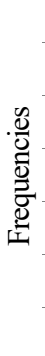 } & $2,5 \mathrm{~Hz}$ & $0,8991(0,0179)$ & $0,9427(0,0049)$ & $1,0021(0,0107)$ & $1,1077(0,0291)$ & $1,0925(0,0029)$ & $1,0738(0,0028)$ & $\mathrm{X}$ \\
\hline & $5 \mathrm{~Hz}$ & $\mathrm{X}$ & $0,9149(0,0074)$ & $0,9519(0,0096)$ & $0,9791(0,0083)$ & $1,0800(0,0070)$ & $1,0509(0,0102)$ & $1,0119(0,0175)$ \\
\hline & $10 \mathrm{~Hz}$ & $\mathrm{X}$ & $0,9640(0,0171)$ & $1,0225(0,0012)$ & $1,0646(0,0138)$ & $1,0180(0,0205)$ & $1,0254(0,0133)$ & $1,0404(0,0029)$ \\
\hline & $20 \mathrm{~Hz}$ & $\mathrm{X}$ & $\mathrm{X}$ & $\mathrm{X}$ & $1,0846(0,0106)$ & $1,0771(0,035)$ & $1,0610(0,0113)$ & $1,0366(0,0083)$ \\
\hline & $49 \mathrm{~Hz}$ & $\mathrm{X}$ & $\mathrm{X}$ & $\mathrm{X}$ & $\mathrm{X}$ & $1,0877(0,0082)$ & $1,0768(0,0035)$ & $1,0280(0,0098)$ \\
\hline & $95 \mathrm{~Hz}$ & $\mathrm{X}$ & $\mathrm{X}$ & $\mathrm{X}$ & $\mathrm{X}$ & $\mathrm{X}$ & $1,1538(0,0109)$ & $1,1165(0,0116)$ \\
\hline & $159,2 \mathrm{~Hz}$ & $\mathrm{X}$ & $\mathrm{X}$ & $\mathrm{X}$ & $\mathrm{X}$ & $\mathrm{X}$ & $1,1361(0,0213)$ & $X$ \\
\hline
\end{tabular}

Table 5. Multiplication Factors for the MPU-6050 (average and standard-deviation values).

\begin{tabular}{|c|c|c|c|c|c|c|c|c|}
\hline & & \multicolumn{7}{|c|}{ Acceleration band } \\
\hline & & $0,02 \mathrm{~g}$ & $0,05 \mathrm{~g}$ & $0,1 \mathrm{~g}$ & $0,2 \mathrm{~g}$ & $0,5 \mathrm{~g}$ & $1 \mathrm{~g}$ & $1,5 \mathrm{~g}$ \\
\hline \multirow{7}{*}{ 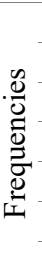 } & $2,5 \mathrm{~Hz}$ & $0,8801(0,0200)$ & $0,9487(0,0013)$ & $0,9912(0,0112)$ & $1,0842(0,0079)$ & $1,0906(0,0013)$ & $1,0813(0,0036)$ & $X$ \\
\hline & $5 \mathrm{~Hz}$ & $X$ & $0,9035(0,0071)$ & $1,0380(0,0253)$ & $1,0245(0,0133)$ & $1,0386(0,0018)$ & $1,0558(0,0055)$ & $1,0587(0,0123)$ \\
\hline & $10 \mathrm{~Hz}$ & $X$ & $1,0287(0,0135)$ & $1,0799(0,0166)$ & $1,0569(0,0233)$ & $1,0486(0,0048)$ & $1,0582(0,0104)$ & $1,0281(0,0237)$ \\
\hline & $20 \mathrm{~Hz}$ & $X$ & $\mathrm{X}$ & $\mathrm{X}$ & $1,0839(0,0145)$ & $1,0304(0,0086)$ & $1,0368(0,0122)$ & $1,0436(0,0168)$ \\
\hline & $49 \mathrm{~Hz}$ & $X$ & $X$ & $\mathrm{X}$ & $\mathrm{X}$ & $1,0781(0,0136)$ & $1,0854(0,0126)$ & $1,0482(0,0128)$ \\
\hline & $95 \mathrm{~Hz}$ & $\mathrm{X}$ & $\mathrm{X}$ & $X$ & $X$ & $X$ & $1,2006(0,0066)$ & $1,1614(0,0178)$ \\
\hline & $159,2 \mathrm{~Hz}$ & $\mathrm{X}$ & $\mathrm{X}$ & $\mathrm{X}$ & $\mathrm{X}$ & $X$ & $1,1980(0,0117)$ & $\mathrm{X}$ \\
\hline
\end{tabular}

Figure 11 depicts the responses at $2.5 \mathrm{~Hz} / 0.02 \mathrm{~g}$ (low signal-to-noise ratio) for all accelerometers in the time domain after applying the multiplication factors. A general good agreement can be seen among the accelerometers' outputs. 


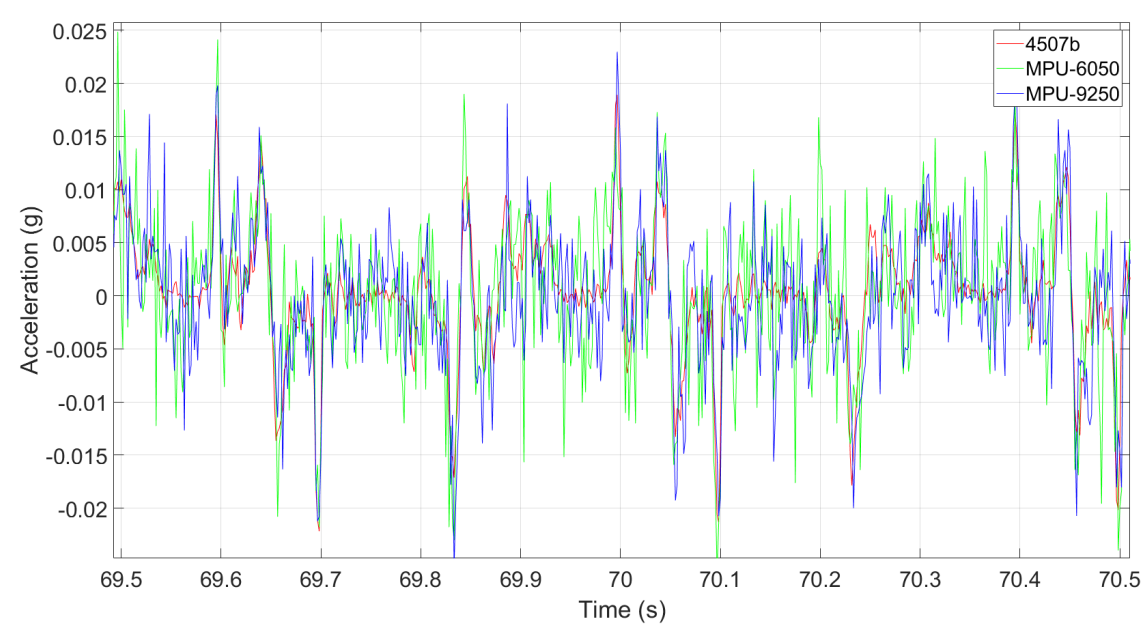

Figure 11. Responses in the time domain using the modulated shaker at 2,5 Hz/0,02g.

Figure 12 shows the $2.5 \mathrm{~Hz} / \mathrm{lg}$ test responses with a high signal-to-noise ratio, where it is possible to observe the vibration behaviors on a scale where the noise is visually imperceptible for the three accelerometers.

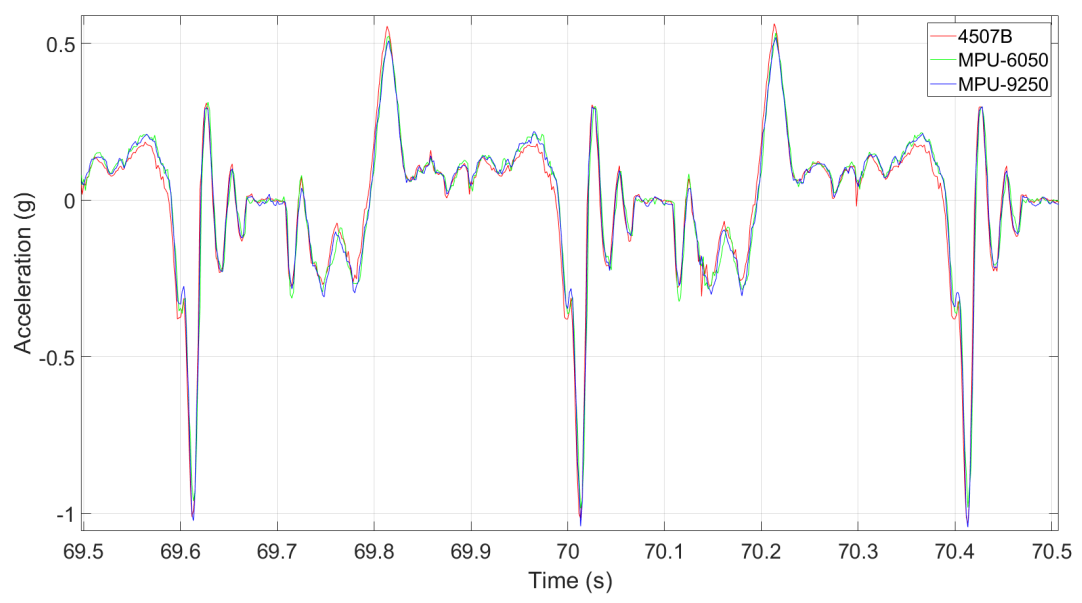

Figure 12. Responses in the time domain using the modulated shaker at $2,5 \mathrm{~Hz} / 1 \mathrm{~g}$.

In summary, from the results obtained, considering a frequency range of $2.5 \mathrm{~Hz}$ to $49 \mathrm{~Hz}$, it is safe to stipulate a margin of error of $10 \%$ between the measurements of the prototypes and the commercial accelerometer. If the frequency range is between $2.5 \mathrm{~Hz}$ and $159.2 \mathrm{~Hz}$, such a margin increases to $20 \%$. These are estimates for the reading errors of the MPU-6050 and MPU-9250 sensors, not including design safety factors, which must be added after considering the margin of error of the accelerometers. If the structural applications allow the margin of error presented in this work and are within the frequency range stipulated for the allowed error, then it is possible to use the MPU-6050 or MPU-9250.

Consequently, the main performance loss of the MPU-6050 (and the MPU-9250) happens in the time domain. Prototype accelerometers are not intended to achieve high-frequency performance, such as the 4507B that works up to $6 \mathrm{kHz}$ with a 10\% margin of error. In this study, it was possible to verify the reliability of the sensors for frequency bands compatible mainly with those found in SHM of civil engineering structures, such as buildings and bridges.

Finally, it is important to emphasize that the multiplication factors yield values that would best fit the signals' trend and not measurement-to-measurement values, which would be very difficult to achieve and not so useful, since in a structural health monitoring system, for example, numerical analyses would treat such peaks as outliers. 


\section{FINAL REMARKS AND CONCLUSIONS}

This paper proposed a low-cost wireless accelerometer for dynamic structural monitoring based on a single-board Raspberry Pi computer. The entire planning, coding, execution, and evaluation process was carried out with the objective of reducing implementation costs while maintaining data's integrity. The main idea of this paper was to present a raw comparison between low cost and commercial accelerometers and show how close, in terms of performance and reliability, the former could get to the latter.

To assess the prototypes performance, several experimental applications were performed using controlled excitations. Firstly, the noise of the sensors at rest were evaluated. Both low-cost sensors presented higher noise values compared to the commercial accelerometer. Thus, for applications where readings are in the range of $0.001 \mathrm{~g}$, prototypes are not indicated. However, for accelerations above $0.01 \mathrm{~g}$, the use of the prototypes becomes viable. Moreover, it is important to keep in mind that no type of signal processing or conditioning (such as filtering, amplification, attenuation, among others) was performed during the acquisition process for both MPUs. If that were the case, such discrepancies would be largely reduced.

When it comes to frequency domain analyses, the prototypes presented remarkably similar performance compared to the commercial accelerometer for the tested frequency range between $2.5 \mathrm{~Hz}$ and $159.2 \mathrm{~Hz}$.

Finally, in the time domain, the prototypes presented their largest differences compared to the commercial accelerometer. This happened since the sensors used in the prototypes do not have a calibration constant, such as the 4507B. However, by evaluating specific multiplication factors, those differences have significantly decreased.

In summary, the proposed prototypes could represent a potential low-cost replacement of conventional monitoring cabled systems or commercial wireless sensors for SHM applications. When it comes to field testing in real large-scale structures, it is imperative to carry out preliminary tests to assess suitable multiplication factors - according to frequency and acceleration ranges - so that the results will represent the structure's behavior properly.

As for further developments, the authors are working on fully wireless sensors using small solar panels as sources of energy and the remote synchronization of several sensors. Moreover, a special focus is being given to embedded data compression techniques along with filtering procedures. Finally, the proposed prototypes will be tested in real civil structures in the near future in applications concerning modal tracking and structural damage identification.

\section{ACKNOWLEDGEMENTS}

The authors would like to thank CAPES (Coordenação de Aperfeiçoamento de Pessoal de Nível Superior - Finance Code 001), CNPq (Conselho Nacional de Desenvolvimento Científico e Tecnológico - Finance Code 304329/2019-3), FAPEMIG (Fundação de Amparo à Pesquisa do Estado de Minas Gerais - Finance Codes PPM-00002-16 / 00001-18), UFJF (Universidade Federal de Juiz de Fora) and PUC-MG (Pontificia Universidade Catolica of Minas Gerais) for the financial support.

\section{REFERENCES}

[1] R. Battista and M. Pfeil, "Reduction of vortex-induced oscillations of Rio-Niterói Bridge by dynamic control devices," J. Wind Eng. Ind. Aerodyn., vol. 84, no. 3, pp. 273-288, 2000, http://dx.doi.org/10.1016/S0167-6105(99)00108-7.

[2] J. Maeck and G. De Roeck, "Description of Z24 benchmark," Mech. Syst. Signal Process., vol. 17, no. 1, pp. 127-131, 2003, http://dx.doi.org/10.1006/mssp.2002.1548.

[3] E. Cachot, T. Vayssade, M. Virlogeux, H. Lancon, Z. Hajar, and C. Servant, "The Millau Viaduct: ten years of structural monitoring," Struct. Eng. Int., vol. 25, no. 4, pp. 375-380, 2015, http://dx.doi.org/10.2749/101686615X14355644770776.

[4] S. Soyoz and M. Q. Feng, "Long-term monitoring and identification of bridge structural parameters," Comput. Aided Civ. Infrastruct. Eng., vol. 24, no. 2, pp. 82-92, 2009, http://dx.doi.org/10.1111/j.1467-8667.2008.00572.x.

[5] A. J. Cardini and J. T. DeWolf, "Long-term structural health monitoring of a multi-girder steel composite bridge using strain data," Struct. Health Monit., vol. 8, no. 1, pp. 47-58, 2009, http://dx.doi.org/10.1177/1475921708094789.

[6] R. Cardoso, A. Cury, and F. Barbosa, "A robust methodology for modal parameters estimation applied to SHM," Mech. Syst. Signal Process., vol. 95, pp. 24-41, 2017, http://dx.doi.org/10.1016/j.ymssp.2017.03.021.

[7] Z. R. Lu and S. S. Law, "Dynamic condition assessment of a cracked beam with the composite element model," Mech. Syst. Signal Process., vol. 23, no. 2, pp. 415-431, 2009, http://dx.doi.org/10.1016/j.ymssp.2008.02.009.

[8] G. D. Ercolani, D. H. Felix, and N. F. Ortega, "Crack detection in prestressed concrete structures by measuring their natural frequencies," J. Civ. Struct. Health Monit., vol. 8, no. 4, pp. 661-671, 2018, http://dx.doi.org/10.1007/s13349-018-0295-2.

[9] I. Z. Mat Darus and M. O. Tokhi, "Soft computing-based active vibration control of a flexible structure," Eng. Appl. Artif. Intell., vol. 18, no. 1, pp. 93-114, 2005, http://dx.doi.org/10.1016/j.engappai.2004.08.017. 
[10] L. Qiusheng, H. Yinghou, Z. Kang, H. Xuliang, H. Yuncheng, and S. Zhenru, "Structural health monitoring for a 600m high skyscraper," Struct. Des. Tall Spec. Build., vol. 27, no. 12, 2018, http://dx.doi.org/10.1002/tal.1490.

[11] D. Ribeiro, J. Leite, N. Pinto, and R. Calçada, "Continuous monitoring of the dynamic behaviour of a high-rise telecommunications tower," Struct. Des. Tall Spec. Build., vol. 28, no. 11, pp. e1621, 2019., http://dx.doi.org/10.1002/tal.1621.

[12] A. Cury, C. C. H. Borges, and F. S. Barbosa, "A two-step technique for damage assessment using numerical and experimental vibration data," Struct. Health Monit., vol. 10, no. 4, pp. 417-428, 2010, http://dx.doi.org/10.1177/1475921710379513.

[13] R. P. Finotti, A. A. Cury, and F. S. Barbosa, "An SHM approach using machine learning and statistical indicators extracted from raw dynamic measurements," Lat. Am. J. Solids Struct., vol. 16, no. 2, pp. e165, 2019, http://dx.doi.org/10.1590/1679-78254942.

[14] R. Cardoso, A. Cury, and F. Barbosa, "Automated real-time damage detection strategy using raw dynamic measurements," Eng. Struct., vol. 196, 2019, http://dx.doi.org/10.1016/j.engstruct.2019.109364.

[15] L. Melo, D. Ribeiro, R. Calçada, and T. Bittencourt, "Validation of a vertical train-track-bridge dynamic interaction model based on limited experimental data," Struct. Infrastruct. Eng., vol. 16, no. 1, pp. 181-201, 2020, http://dx.doi.org/10.1080/15732479.2019.1605394.

[16] M. A. Torres and S. E. Ruiz, "Structural reliability evaluation considering capacity degradation over time," Eng. Struct., vol. 29, no. 9, pp. 2183-2192, 2007., http://dx.doi.org/10.1016/j.engstruct.2006.11.014.

[17] S. A. Sindhu and C. A. Nirrmala, "Structural health monitoring using wireless sensor network," Int. J. Emerg. Technol. Comput. Sci. Electron., vol. 13, no. 4, pp. 319-322, 2015.

[18] S. Pandey, M. Haider, and N. Uddin, "Design and implementation of a low-cost wireless platform for remote bridge health monitoring," Int. J. Emerg. Technol. Adv. Eng., vol. 6, no. 6, pp. 57-62, 2016.

[19] A. B. Noel, A. Abdaoui, T. Elfouly, M. H. Ahmed, A. Badawy, and M. Shehata, "Structural health monitoring using wireless sensor networks: a comprehensive survey," IEEE Comm. Surv. and Tutor., vol. 19, no. 3, pp. 1403-1423, 2016, http://dx.doi.org/10.1109/COMST.2017.2691551.

[20] E. Sazonov, Haodong Li, D. Curry, and P. Pillay, "Self-powered sensors for monitoring of highway bridges," IEEE Sens. J., vol. 9, no. 11, pp. 1422-1429, 2009, http://dx.doi.org/10.1109/JSEN.2009.2019333.

[21] N. Xu et al., "A wireless sensor network for structural monitoring," in Proc. 2nd Int. Conf. Embed. Netw. Sensor Syst., SenSys '04, 2004, pp. 13-24, http://dx.doi.org/10.1145/1031495.1031498.

[22] M. Varanis, A. L. Silva, P. H. A. Brunetto, and R. F. Gregolin, "Instrumentation for mechanical vibrations analysis in the time domain and frequency domain using the Arduino platform," Rev. Bras. Ensino Fis., vol. 38, no. 1, 2016, http://dx.doi.org/10.1590/S1806-11173812063.

[23] A. Abdelgawad and K. Yelamarthi, "Internet of Things (IoT) platform for structure health monitoring," Wirel. Commun. Mob. Comput., vol. 2017, pp. 1-10, 2017, http://dx.doi.org/10.1155/2017/6560797.

[24] A. Afsana, S. Anbarasi, P. Deepa, R. Ghaveya, and D. Marugesan, "Wireless sensor network based crack detection on concrete bridges/buildings," Int. J. Eng. Trends Technol., vol. 57, no. 2, pp. 54-58, 2018, http://dx.doi.org/10.14445/22315381/IJETTV57P211.

[25] P. Shachi and S. Manjunatha, "Automatic bridge health monitoring system using wireless sensors," Int. J. Sci. Res., vol. 6, no. 6, pp. 2472-2475, 2015.

[26] V. Chandankhede, "Health monitoring of highway bridges using wireless sensor networks," Int. J. Adv. Res. Electron. Commun. Eng., vol. 6, no. 8, pp. 927-930, 2017.

[27] Raspbian. https://www.raspberrypi.org/documentation/raspbian/ (accessed Apr. 21, 2020).

[28] Putty. https://www.chiark.greenend.org.uk/ sgtatham/putty/docs.html (accessed Apr. 21, 2020).

[29] WinSCP. https://winscp.net/eng/docs/start (accessed Apr. 21, 2020).

Author contributions: ET: Conceptualization, Data curation, Formal analysis, Investigation, Methodology, Writing-original draft, Writing-review \& editing. AC: Conceptualization, Data curation, Formal analysis, Funding acquisition, Investigation,

Methodology, Project administration, Supervision, Validation, Writing-review \& editing. JL: Conceptualization, Data curation, Formal analysis,

Funding acquisition, Investigation,

Methodology, Resources, Supervision.

Editors: Tulio Nogueira Bittencourt, José Luiz Antunes de Oliveira e Sousa, Guilherme Aris Parsekian. 